\section{Da evidência à ação: desafios do Sistema Único de Saúde para ofertar a profilaxia pré-exposição sexual (PrEP) ao HIV às pessoas em maior vulnerabilidade}

\author{
From evidence to action: challenges for the \\ Brazilian Unified National Health System in \\ offering pre-exposure prophylaxis (PrEP) for \\ HIV to persons with the greatest vulnerability \\ De la evidencia a la acción: desafíos del Sistema \\ Único de Salud para ofrecer la profilaxis pre- \\ exposición sexual (PrEP) al VIH a personas \\ con mayor vulnerabilidad
}

Eliana Miura Zucchi 1 Alexandre Grangeiro 2 Dulce Ferraz 3 Thiago Félix Pinheiro 2 Tatianna Alencar 4 Laura Ferguson 5 Denize Lotufo Estevam 6 Rosemeire Munhoz 6 Equipe do Estudo Combina! 7

doi: 10.1590/0102-311X00206617

\title{
Resumo
}

A profilaxia pré-exposição sexual (PrEP) tem sido considerada estratégica e promissora no controle da epidemia de HIV globalmente. Contudo, faz-se necessário transpor o conhecimento acumulado pelos estudos de eficácia e demonstrativos à realidade dos serviços e das populações mais vulneráveis à infecção, de forma a alcançar uma ampla cobertura da PrEP. Propõe-se uma reflexão sobre tal desafio enfocando duas dimensões: os usuários da profilaxia, com ênfase nos contextos de práticas e de potencial exposição ao HIV; as vantagens comparativas da profilaxia em relação aos demais métodos e os desafios para um uso protetivo e seguro; e os serviços, considerando os princípios organizativos que poderão conferir maior êxito na oferta e na incorporação da PrEP, no bojo das estratégias de prevenção combinada. Foram analisados como alguns princípios - singularidade no cuidado, autonomia para escolha e não hierarquização dos métodos, gestão de risco sexual e flexibilidade de agendamento e complementaridade de uma equipe multiprofissional - podem favorecer a organização do serviço e do cuidado, facilitando a vinculação e retenção dos usuários. Teceram-se algumas considerações acerca de uma relativa incompatibilidade entre a estrutura existente nos serviços e as diretrizes do Ministério da Saúde para oferta de PrEP. Concluiu-se que o maior êxito da PrEP como política pública de saúde depende de dois aspectos centrais: assegurar que os serviços sejam ambientes culturalmente diversos e livres de discriminação e a intensificação das intervenções comunitárias, incluindo as redes sociais, de forma a reduzir iniquidades no acesso aos serviços e à PrEP.

Profilaxia Pré-Exposição; HIV; Saúde Sexual; Serviços de Saúde

\section{Correspondência}

E. M. Zucchi

Programa de Pós-graduação em Saúde Coletiva, Universidade Católica de Santos.

Av. Conselheiro Nébias 300, Santos, SP 11015-001, Brasil. eliana.zucchi@uol.com.br

1 Programa de Pós-graduação em Saúde Coletiva, Universidade Católica de Santos, Santos, Brasil.

2 Faculdade de Medicina, Universidade de São Paulo, São Paulo, Brasil.

3 Escola Fiocruz de Governo, Fundação Oswaldo Cruz, Brasília, Brasil.

4 Departamento de Vigilância, Prevenção e Controle das Infecções Sexualmente Transmissiveis, do HIV/Aids e das Hepatites Virais, Ministério da Saúde, Brasília, Brasil.

${ }^{5}$ Keck School of Medicine, University of Southern California, Los Angeles, U.S.A.

6 Centro de Referência e Treinamento em DST/AIDS-SP,

Secretaria de Estado de Saúde de São Paulo, São Paulo, Brasil.

7 Outros membros listados ao final do artigo. 


\section{Introdução}

A profilaxia pré-exposição sexual (PrEP) está no centro do debate sobre as novas possibilidades de prevenção do HIV, aumentando o otimismo acerca do controle da epidemia globalmente. Caracteriza-se pelo uso cotidiano da combinação de dois antirretrovirais (tenofovir associado à entricitabina - TDF/FTC) antecedendo as práticas sexuais, com grau de proteção de 96\% (90\% a > 99\%) nas relações anais, quando utilizada por pelo menos quatro dias na semana 1. Em tese, a disponibilidade de distintos métodos preventivos tem maior potencial para abranger diferentes grupos sociais, permitindo escolhas preventivas de acordo com contextos, necessidades e preferências 2 . Também chamada de "prevenção combinada", trata-se de uma estratégia que propõe a oferta de novos métodos preventivos - profilaxias pré- e pós-exposição sexual (PrEP e PEP) - e dos métodos clássicos (preservativos masculino e feminino, promoção de práticas não penetrativas e uso da testagem anti-HIV para acordos sexuais), combinada com intervenções comportamentais e estruturais reconhecidamente efetivas para o enfrentamento da epidemia 3,4 .

Estudos clínicos e demonstrativos globalmente contribuíram para gerar um relativo consenso acerca dos benefícios individuais e populacionais da PrEP, assim como para estabelecer parâmetros clínicos de uso seguro 5. Porém, persistem indagações sobre os efeitos compensatórios da PrEP nas práticas sexuais, a relação entre vulnerabilidade social e adesão e os entraves operacionais para assegurar acesso amplo, seguro e efetivo à profilaxia 5. Tais questões têm dificultado a expansão da PrEP no mundo, especialmente nos países de média e baixa renda 6,7 . A consequência é que baixas taxas de cobertura minimizariam o potencial impacto da PrEP na epidemia de HIV. Nos Estados Unidos, por exemplo, modelagem matemática estimou uma redução de 1/3 das novas infecções em homossexuais somente se o uso de PrEP atingir, por um período de dez anos, $40 \%$ dessa população 8 .

No Brasil, os métodos preventivos baseados em antirretrovirais (ARV) têm sido gradualmente incorporados ao Sistema Único de Saúde (SUS). A oferta da PrEP foi iniciada ao final de 20179 para grupos com maior risco de infecção. A oferta pública foi antecedida por estudos demonstrativos. O PrEP-Brasil, realizado entre 2014-2015 com 450 homens que fazem sexo com homens (HSH) e mulheres transexuais, mostrou uma taxa de aceitação da PrEP de 60\% e estabilidade temporal no relato de número de parceiros, de relações sexuais desprotegidas e da incidência de infecções sexualmente transmissíveis (IST). As taxas variaram, ao longo do tempo, entre 8\% e 7,7\% para clamídia retal e entre 4,9\% e 3,7\% para gonorreia retal 10. No Estudo Combina! 11, realizado com homens e mulheres com alta exposição ao HIV, a oferta de PrEP foi iniciada em 2016 e envolveu, até setembro de 2017, cerca de 380 indivíduos. Análises iniciais mostraram concentração da busca da profilaxia por homens gays ou HSH (91\%), com elevado nível socioeconômico (61\% com superior completo ou incompleto), tendo seguro privado de saúde (51\%) e alta frequência de parcerias ocasionais (90\%), encontradas sobretudo pela Internet (84\%). Taxas elevadas (24\%) de IST, bem como de realização de PEP foram relatadas para os seis meses antecedentes à inclusão no estudo.

$\mathrm{O}$ atual desafio, nesse contexto, é transpor o conhecimento adquirido em estudos de eficácia e demonstrativos à realidade dos serviços e das populações mais vulneráveis à infecção, de forma a alcançar uma ampla cobertura da PrEP. O presente ensaio objetiva, assim, refletir sobre esse desafio que enfoca duas dimensões: os usuários da profilaxia, com ênfase nos contextos de práticas e de potencial exposição ao HIV, as vantagens comparativas da profilaxia em relação aos demais métodos e os desafios para um uso protetivo e seguro; e os serviços, considerando os princípios organizativos que poderão conferir maior êxito na oferta e na incorporação da PrEP, no bojo das estratégias de prevenção combinada. Discutiram-se, ainda, as articulações entre ações programáticas e estruturais para a ampla incorporação da PrEP. Para tanto, articularam-se conhecimentos adquiridos na implantação da PrEP nos cinco serviços participantes do Estudo Combina!, na revisão de normas para uso profilaxia de diferentes instituições e na literatura científica. 


\section{Considerações acerca da PrEP: um olhar para o usuário, seu contexto e necessidades}

\section{A indicação da PrEP}

Estudos têm mostrado que os benefícios individuais e populacionais da PrEP aumentam conforme o risco de infecção dos usuários da profilaxia 5 . Assim, indivíduos sob baixo risco de infecção obteriam, em tese, maior benefício com outros métodos preventivos (preservativos, PEP, teste anti-HIV) e poderiam estar expostos a riscos desnecessários devido a efeitos adversos e/ou a menor motivação para adesão.

Com base nisso, diretrizes de diferentes países e instituições têm restringido a recomendação de PrEP às populações sob alto risco de infecção. Nas diretrizes da Organização Mundial da Saúde (OMS) de 2015 12, alto risco é definido como incidência do HIV igual ou superior a 3\%. No Brasil, em 2017, diretrizes do Ministério da Saúde priorizaram quatro segmentos: gays e outros HSH; pessoas transexuais; trabalhadores/as do sexo; e parcerias sorodiferentes, desde que tenham tido, nos últimos seis meses, relações anais ou vaginais sem preservativo, episódios de IST recorrentes ou uso repetido de PEP 13. Pessoas de segmentos com maior vulnerabilidade social e dificuldades de acesso aos serviços, notadamente pessoas negras, adolescentes, usuárias de drogas e privadas de liberdade figuram como prioritárias nos documentos de prevenção combinada, mas não há ações específicas relativas à oferta de PrEP para elas.

As vantagens da PrEP podem ser maiores a depender dos aspectos contextuais, das circunstâncias que envolvem as práticas sexuais e das possibilidades concretas de adotar estratégias preventivas. A possibilidade de o uso ser planejado antes da relação sexual e com relativa independência de negociação das parcerias, aumenta o grau de proteção em situações em que a negociação preventiva é dificultada ou indesejada, por exemplo, quando o sexo ocorre concomitantemente ao uso de álcool e drogas em locais públicos ou envolvendo práticas estigmatizadas. Pode facilitar, também, a prevenção em situações em que a autonomia para negociar o uso de outros métodos é limitada, como relações em contextos de violência ou de trabalho sexual. Cabe lembrar que questões como dificuldades ou impossibilidade de negociação, uso de álcool e droga e a violência são elementos presentes em diferentes cenários sexuais e são reconhecidos como importantes barreiras à consecução da prevenção 14,15.

A PrEP é também uma alternativa para pessoas que não conseguem ou não desejam usar os métodos clássicos. No Brasil, embora 94\% reconheçam o preservativo como a melhor forma de evitar o HIV, apenas 19,9\% afirmam utilizá-lo com parcerias fixas e 54,9\% com parcerias casuais 16 . Essa pode ser outra vantagem da PrEP, que oferece proteção concomitantemente nas relações estáveis e ocasionais.

A PrEP pode ainda ser usada de forma conjugada com outros métodos preventivos, por pessoas que usam preservativos de forma inconsistente ou que têm parcerias com risco substancial de infecção ou soropositivas, com carga viral detectável ou desconhecida.

Na definição de estratégias para a oferta da PrEP, há que se ter em conta que pertencer a determinados segmentos não implica, obrigatoriamente, maior risco de infecção. Isso impõe aos serviços e profissionais de saúde o desafio de transpor a lógica populacional que fundamentou as diretrizes, para o âmbito do indivíduo, de modo que a avaliação das práticas sexuais e do risco não ocorra de forma simplificadora. A exposição ao HIV e as possibilidades de proteger-se dela são conformadas por várias dimensões e, portanto, devem ser avaliadas pela compreensão articulada de aspectos individuais, sociais e programáticos 17 . Trata-se, então, de empreender uma abordagem dialógica, com base no reconhecimento de que a experiência de cada indivíduo congrega, de modo singular, características do(s) grupo(s) a que pertence, do lugar e do momento histórico em que vive.

Uma avaliação abrangente do risco de infecção e da indicação de PrEP deveria perpassar três aspectos:

(1) Práticas sexuais: sobressaem como indicativas para PrEP as penetrações anal e vaginal sem preservativo, com maior chance de infecção para a primeira e para quem é penetrado, embora também haja risco significativo para quem penetra 18 . Ejaculação no ânus e na vagina aumentam o risco, assim como penetração com punho ou objetos que produzam fissuras. O risco no sexo oral é baixo, mas 
pode aumentar com ejaculação na boca e ocorrência de feridas ou sangramento ${ }^{19}$. Ter histórico de IST, além de aumentar a chance de infecção por HIV, indica práticas sexuais desprotegidas, assim como a busca repetida de PEP.

(2) Tipo de parcerias sexuais: as eventuais indicam predominantemente situações de maior exposição ao HIV. Parcerias estáveis, por sua vez, estão associadas a pelo menos $1 / 3$ das infecções por HIV, incluindo as que ocorrem em gays e outros HSH em contextos de epidemia concentrada 20. Tais parcerias estáveis estão associadas à maior frequência de relações sexuais, de práticas penetrativas e de não uso de métodos preventivos. Relações entre parcerias sorodiferentes têm suscitado discussões. Por um lado, há evidências consistentes de que pessoas com HIV com supressão da replicação viral por tratamento com ARV possuem risco desprezível de transmitir o vírus 21. Por outro lado, há situações concretas em que pessoas soronegativas desconhecem informações do tratamento da parceria soropositiva e podem se encontrar em situação desfavorável para negociar a prevenção.

(3) Contextos em que as práticas sexuais ocorrem: sob uso de álcool e drogas e as consequentes alterações da percepção e do controle físico e emocional; o estigma das práticas não vinculadas diretamente à reprodução, que induz ao sexo em ambientes alternativos e sustenta a violência homofóbica e sexista; as condições do trabalho sexual, frequentemente marcadas pela precariedade em razão da ilegalidade das casas de prostituição.

Cabe ressaltar, ainda, que as situações de exposição ao HIV são transitórias ao longo da vida, definindo momentos em que a PrEP é necessária e outros em que não, a depender da prática do indivíduo. Isso requer avaliações contínuas dos profissionais de saúde de forma a oportunizar ou propor a suspensão do uso da PrEP de modo adequado, o que insere o cuidado do usuário de PrEP em um processo dinâmico e contínuo que deve se basear, primordialmente, no diálogo sobre a adequação às necessidades de autocuidado que esse método preventivo oferece no contexto das práticas sexuais do indivíduo. É importante chamar a atenção para o fato de que, independentemente da possibilidade de uma transitoriedade nas práticas individuais, uma parcela da população pode apresentar uma frequência de potencial exposição que não motiva o indivíduo a querer o uso contínuo da PrEP, ou mesmo que justifique plenamente a prescrição contínua de PrEP. Nesses casos, é necessário discutir a possibilidade de uso consistente de outros métodos preventivos. Além disso, alternativa seria o esquema de tomada intermitente da PrEP, a chamada "PrEP sob demanda”. Essa estratégia teve sua eficácia mostrada no estudo Ipergay 22, que observou uma redução de risco de infecção pelo HIV de $86 \%$ em HSH que usaram dois comprimidos de TDF/FTC entre 24 e 2 horas antes da relação sexual e dois comprimidos após (24 horas e 48 horas), sem riscos adicionais de eventos adversos ou de resistência viral. Essa estratégia, no entanto, não está regulamentada no Brasil por falta de estudos demonstrativos para comprovar sua efetividade na realidade nacional.

\section{Padrão de uso}

Estudos têm indicado maior descontinuidade da PrEP no período inicial de uso. Em gays e HSH estadunidenses acompanhados em clínicas de IST, cerca de 22\% abandonaram a profilaxia após um ano ${ }^{23}$. Em outro estudo, no mesmo país, esse percentual foi $60 \%$, no sexto mês ${ }^{3}$. No Estudo Combina!, a taxa de abandono do uso da profilaxia após seis meses de seguimento foi pouco superior a $12 \%$ e não esteve relacionada ao menor risco de infecção ou de nível social, o que levou a supor que as práticas sexuais que justificaram a indicação de PrEP persistiram após a descontinuidade do uso.

Há também a possibilidade de parcelas de usuários de PrEP apresentarem um padrão de uso marcado por intervalos de não uso, o que pode ser positivo se a interrupção estiver associada ao uso de outros métodos ou à redução significativa do risco de infecção, como comentado anteriormente. Entretanto, alguns estudos mostram que proporções significativas das infecções por HIV ocorreram durante períodos de interrupção, que foram motivados, entre outros aspectos, por algum agravo à saúde 24,25. No estudo PROUD, um ensaio aberto com gays e HSH ingleses, 5\% dos voluntários interromperam a PrEP por efeitos adversos, como náuseas e dores de cabeça e articulares, tendo a quase totalidade retornado posteriormente 26 .

Se forem considerados períodos mais longos, outros fatores poderão dificultar o uso contínuo da profilaxia, como a eventual fadiga de uso do medicamento, a ocorrência de efeitos adversos em longo prazo e dificuldades de cumprir um protocolo clínico que exige frequentes visitas aos serviços. No 
estudo Kaiser, realizado em São Francisco, Estados Unidos, este último ponto foi um dos principais fatores para não iniciar a PrEP 27.

\section{Adesão}

A adesão é determinante da efetividade da PrEP. A frequência de uso de tenofovir/entrecitabina por pelo menos quatro dias da semana tem sido maior na vida real 23,28 do que nos estudos clínicos de eficácia, provavelmente porque os usuários escolheram esse método e sabem que ele é comprovadamente eficaz.

No contexto brasileiro, o estudo demonstrativo PrEP-Brasil mostrou a factibilidade de obter taxas de adesões próximas a $80 \%$ entre gays e HSH e mulheres transexuais 10. No Estudo Combina!, nos seis primeiros meses, cerca de $75 \%$ dos participantes retiraram o medicamento no tempo adequado para manter o uso regular. Porém, a maior vulnerabilidade social parece influenciar negativamente a adesão. No PrEP-Brasil, ser transexual, jovem e ter menor nível socioeconômico aumentou a chance de não adesão. Similarmente, em dois estudos nos Estados Unidos foi encontrada pior adesão entre gays e HSH negros e em indivíduos como menor percepção do risco de infecção 3,10,23,29. Ainda, em estudo demonstrativo com 200 adolescentes gays e outros HSH estadunidenses, as condições de acesso ou de inadequação dos serviços às características desse público colaboraram para pior adesão 30 .

A oferta da PrEP demanda, portanto, atenção especial à adesão, tanto no sentido de desenvolver estratégias para aumentar o vínculo com os serviços, quanto para identificar indivíduos mais vulneráveis à não adesão e apoiá-los no uso cotidiano do medicamento. O Quadro 1 sintetiza estratégias que têm auxiliado na melhor adesão.

\section{Quadro 1}

Orientações a usuários(as) de profilaxia pré-exposição sexual (PrEP).

\begin{tabular}{|c|c|}
\hline $\begin{array}{l}\text { Tempo para se alcançar proteção após início } \\
\text { do uso da medicação }\end{array}$ & São necessários 7 e 21 dias para proteção na relações anais e vaginais, respectivamente. \\
\hline Frequência de tomada do medicamento & $\begin{array}{l}\text { Recomenda-se que o medicamento seja tomado diariamente, independentemente do horário. } \\
\text { Associar a tomada a alguma atividade realizada cotidianamente (ao tomar café da manhã, } \\
\text { escovar os dentes, estudar, entre outras) reduz a chance de esquecimento. A concentração } \\
\text { da droga no organismo é mantida com quatro ou mais dias de uso semanal. Pular um ou } \\
\text { dois dias de uso do medicamento não compromete significativamente o grau de proteção da } \\
\text { profilaxia. Nesse caso, a melhor indicação é continuar o uso regular no dia posterior e jamais } \\
\text { tomar dois comprimidos em um mesmo dia. }\end{array}$ \\
\hline $\begin{array}{l}\text { Tempo considerado como interrupção/ } \\
\text { finalização da profilaxia }\end{array}$ & $\begin{array}{l}\text { A redução dos níveis ideais de proteção ocorre a partir de três dias contínuos sem o uso do } \\
\text { medicamento. Nesse caso, para reintrodução da PrEP, é recomendado seguir os mesmos } \\
\text { procedimentos de início de profilaxia, com a realização de teste anti-HIV e a investigação da } \\
\text { presença de sinais e sintomas de infecção aguda e IST. Para indivíduos que relatam práticas } \\
\text { sexuais penetrativas com risco de exposição ao HIV nas últimas } 72 \text { horas, considerar a } \\
\text { indicação de PEP. }\end{array}$ \\
\hline Efeitos adversos & $\begin{array}{l}\text { Os efeitos tendem a ser brandos e a desaparecer com menos de um mês de uso. Os mais } \\
\text { comuns são náusea, enjoo, diarreia e gases. }\end{array}$ \\
\hline Uso concomitante de álcool ou outras drogas & Não há contraindicação no uso conjunto de PrEP e álcool ou outras drogas. \\
\hline
\end{tabular}

IST: infecções sexualmente transmissíveis; PEP: profilaxia pós-exposição sexual.

Fonte: Ministério da Saúde 13. 


\section{IST e desinibição sexual}

Embora estudos indiquem que após o início da PrEP houve, na maior parte das vezes, redução ou manutenção das práticas sexuais com maior risco 25 , persistem dúvidas se grupos específicos apresentariam um efeito de desinibição das práticas sexuais. Em estudo demonstrativo com gays e HSH em Providence, Estados Unidos, o número total de parceiros após seis meses de PrEP não se alterou, mas houve um aumento médio de 1,3 vez no número de parcerias com relações anais sem preservativos 31 . Já no estudo Kaiser, a desinibição pode ter ocorrido especificamente nos 11\% e 3\% que relataram, após seis meses, aumento do número de parceiros e de relações anais desprotegidas 27 . No estudo PROUD, uma eventual desinibição pode ter ocorrido naqueles com dez ou mais parceiros com relações sexuais sem o preservativo. Nesse grupo, após um ano, ocorreu aumento do número de parceiros em geral 26. No estudo clínico de eficácia Ipergay, com gays e outros HSH franceses e canadenses, foi verificada maior redução do número de parceiros no grupo que não utilizava PrEP, quando comparados aos usuários de PrEP 22.

Neste mesmo sentido, a relação PrEP e IST tem suscitado interrogações. Evidências sugerem que a frequência de IST não aumenta ao longo do uso de PrEP, mas tende a ser significativamente elevada, além de ser maior do que em não usuários de PrEP. No estudo Iprex, no momento de início da PrEP, a prevalência de sífilis foi cerca de $14 \%$, com incidência de 7,3 casos por 100 pessoas/ano ao longo do tempo. Entre os fatores preditores, estiveram relações anais desprotegidas, presença de herpes e diagnóstico de sífilis antes de PrEP. A incidência de sífilis não variou entre os grupos com ou sem PrEP 32. No estudo Kaiser, a incidência de IST após 12 meses de PrEP foi 50\%, sendo 33\% clamídia, 28\% gonorreia e 5,5\% sífilis. Essas taxas permaneceram altas e estáveis no tempo 27. Também nos Estados Unidos, estudo demonstrativo com gays e HSH e mulheres transgênero em serviços de três cidades mostrou incidência de IST de $90 \%$ ao ano, 48\% para clamídia e $43 \%$ para gonorreia. Novamente, as taxas não variaram no tempo 23 . Independentemente de os estudos não serem conclusivos em relação ao aumento na incidência de IST, é importante destacar que seu diagnóstico deveria ser justamente a porta de entrada para a PrEP 33.

Além disso, há de se reconhecer que IST como sífilis, gonorreia resistente e clamídia 26,34 têm aumentado em diferentes países e regiões do mundo, independentemente dos novos métodos preventivos do HIV. Ainda que alguns estudos chamem a atenção para a necessidade de aprofundar a relação entre a ocorrência de IST e a ampliação do uso de métodos que prescindem do preservativo, como a testagem para acordos sexuais 35 , o aumento da investigação permitirá o diagnóstico, tratamento e, consequentemente, a interrupção precoce da cadeia de transmissão da IST e a redução da gravidade da doença individualmente.

Adicionalmente a isso, é necessário aprimorar estratégias comunitárias de prevenção das IST, facilitar o diagnóstico e tratamento em outros serviços e estratégias de saúde.

\section{Estigma}

O estigma da AIDS pode estar presente na experiência de pessoas que usam PrEP de diferentes maneiras, interferindo negativamente na procura e na adesão à profilaxia 36,37, assim como tornando essas pessoas mais suscetíveis à violência e discriminação 38 .

Portar e tomar medicamentos antirretrovirais pode fazer com que os usuários de PrEP sejam confundidos com pessoas com HIV, resultando em discriminação, como a relatada por um voluntário do Estudo Combina!, que teve dificuldades em uma alfândega por ter sido identificado como infectado pelo HIV, devido aos antirretrovirais que portava. Além disso, a percepção da PrEP como um método "essencialmente gay" pode limitar o uso em outros grupos, como prostitutas e usuários de droga. Finalmente, a associação da PrEP à promiscuidade e irresponsabilidade, sintetizados na expressão em inglês truvada whores (que veicula o estereótipo daqueles que encontram na PrEP um meio de empreender comportamentos de risco) pode resultar na estigmatização das pessoas que escolhem o método, tanto por parte de amigos e parceiros sexuais quanto de profissionais de saúde 39.

Diante dessas situações, a literatura tem debatido a necessidade de enfrentar a estigmatização de usuários de PrEP, valorizando-a como um método associado ao maior prazer, sem que isso seja julgado moralmente como uma escolha inferior aos demais métodos 39 . 
Nesse sentido, dificuldades a serem enfrentadas no uso da PrEP podem incluir a discriminação de pessoas que não se enquadram à heteronormatividade, a culpabilização de pessoas com práticas anais e o medo de pessoas que "transmitiriam" intencionalmente o HIV. Assim, a escolha do uso da PrEP e a adesão ao medicamento dependem, também, de estratégias para o indivíduo lidar com essas questões, bem como iniciativas para mudar no plano simbólico o significado de "quem" e "para quê" se usa PrEP.

\section{Relação entre serviços de saúde e usuários de PrEP}

A organização dos serviços, o tipo de abordagem preventiva que realizam e formação dos profissionais são essenciais para ampliar o acesso e promover a retenção dos usuários de PrEP. A literatura indica que a postura dos profissionais de saúde influencia diretamente a escolha da PrEP, facilitando ou dificultando o acesso, que os grupos com maior potencial de usar a PrEP não costumam frequentar serviços de saúde e que comparecem a eles de forma irregular, mesmo quando estão vinculados. Nesse contexto, a predominância do caráter normativo e prescritivo das ações preventivas nos serviços, com abordagens tecnicistas que não permitem apreender as dimensões contextuais e práticas que caracterizam o modo como os indivíduos vivem suas relações, sua sexualidade e cuidado em saúde 40 , é um desafio a ser enfrentado na implantação da PrEP.

A racionalidade técnica, referente ao sentido instrumental da ação baseada em saberes e artefatos científicos, é indispensável à ação em saúde. Todavia, é preciso que nela sejam também contempladas as implicações simbólicas, relacionais e materiais dos cuidados em saúde em lugar de tratá-las como algo externo à tecnicalidade da ação ${ }^{41}$, permitindo assim compreender os significados que os métodos de prevenção assumem na vida cotidiana dos indivíduos.

No caso do preservativo, por exemplo, é amplamente conhecida a associação do significado da adoção do método com aspectos como confiança, fidelidade, diálogo ou companheirismo entre as parcerias 42. No caso da PrEP, a escolha e a adesão ao método podem estar associadas a aspectos objetivos, como os relacionados à segurança do método e a disponibilidade para visitas periódicas ao serviço, mas também podem ser perpassadas pela subjetividade, como a expectativa de diminuir o medo de se infectar e a disposição para lidar com eventuais efeitos adversos e com o estigma associado aos ARV.

No que se refere à singularidade do cuidado, cada indivíduo possui necessidades conformadas pelo contexto em que vive, pelas percepções que tem do risco e do HIV, pelas relações sexuais que experimenta e por aspectos estruturais, como acesso à renda e ao trabalho, que, em seu conjunto, influenciarão na escolha, no uso do método e na vinculação com o serviço. Isso conduz a abordagens que tomam o indivíduo e as suas necessidades como eixos do cuidado que terão maior chance de êxito. Desse modo, as propostas da clínica ampliada e do projeto terapêutico singular ${ }^{43}$ poderiam ser pensadas para a abordagem preventiva. Similarmente, as técnicas de acolhimento e aconselhamento, largamente disseminadas no campo do HIV, são ferramentas úteis para avaliar risco e vulnerabilidade nas práticas sexuais, subsidiar as escolhas do método e apoiar na adesão ao medicamento e ao seguimento clínico. É preciso, porém, superar a tendência atual de aplicação dessas técnicas, que tem se resumido à recomendação normativa dos métodos em lugar de uma abordagem dialógica em que o usuário é sujeito na definição de planos preventivos 44 .

Por outro lado, a autonomia para escolha e não hierarquização dos métodos pressupõe o reconhecimento de que o maior grau de proteção de qualquer método preventivo é obtido quando utilizado corretamente, nas situações apropriadas e de forma consistente e duradora 2. São fatores que dependem, em grande parte, do indivíduo, dos seus valores e do contexto em que as práticas sexuais ocorrem. Isso leva ao reconhecimento de dois aspectos: primeiro, do ponto de vista da efetividade, não há uma hierarquia de métodos que os serviços ou os profissionais de saúde possam estabelecer a priori; segundo, que o melhor método é aquele que o indivíduo escolhe, orientado por informações cientificamente embasadas, mas de forma autônoma, por ser o mais apropriado às suas necessidades e ao grau de proteção que deseja e às possibilidades que dispõe de utilizá-lo correta e consistentemente ao longo do tempo. Portanto, cada método só tem suas especificidades e vantagens comparativas aos demais (Quadro 2), a depender do contexto e das necessidades do indivíduo. Por exemplo, práticas não penetrativas são mais adequadas àqueles que não privilegiam fortemente práticas genitais, que são mais frequentes nas cenas homossexuais. O teste anti-HIV, visando à prevenção, pode ser alternativa 


\section{Quadro 2}

Métodos preventivos às evidências do grau de proteção.

\begin{tabular}{|c|c|c|c|c|}
\hline $\begin{array}{l}\text { Método } \\
\text { preventivo }\end{array}$ & Uso & Proteção & Vantagens & Desvantagens \\
\hline $\begin{array}{l}\text { Profilaxia pré- } \\
\text { exposição (PrEP) }\end{array}$ & $\begin{array}{l}\text { Uso diário do TDF } \\
\text { ou TDF/FTC. } \\
\text { Uso antes e após } \\
\text { as relações sexuais } \\
\text { (sob demanda). }\end{array}$ & $\begin{array}{l}\text { Em ensaios clínicos, a } \\
\text { proteção global foi de } \\
51 \% 50 \text {. } \\
\text { Em estudos abertos } \\
\text { pós-ensaios clínicos, } \\
\text { o grau de proteção } \\
\text { foi de } 86 \% 26 \text {. } \\
\text { Não há infecções } \\
\text { relatadas para uso } \\
\text { igual ou superior } \\
\text { a quatro dias por } \\
\text { semana } 25 \text {. }\end{array}$ & $\begin{array}{c}\text { Pessoas que conhecem têm } \\
\text { maior risco de exposição e optam } \\
\text { por PrEP podem ter motivações } \\
\text { adicionais para adesão } 25 \text {. } \\
\text { Alta aceitação naqueles que } \\
\text { já utilizaram (65\%) e baixa na } \\
\text { população geral (incertezas sobre } \\
\text { o método, receio de eventos } \\
\text { adversos e baixa percepção do } \\
\text { risco) } 25 \text {. } \\
\text { Ausência de associação entre } \\
\text { adesão e uso de álcool } \\
\text { e droga } 25 \text {. }\end{array}$ & $\begin{array}{l}\text { Dois estudos em mulheres foram } \\
\text { interrompidos por não mostrarem } \\
\text { proteção (baixa adesão, menor } \\
\text { proteção nas relações vaginais, maior } \\
\text { número de processos inflamatórios } \\
\text { na vagina) } 51,52 \text {. } \\
\text { Intercalação de períodos de uso } \\
\text { intenso e não uso (decisão do } \\
\text { indivíduo, eventos adversos e } \\
\text { comorbidades), com as infecções } \\
\text { ocorrendo nessa fase } 25 \text {. } \\
\text { Custos elevados limitam o acesso e a } \\
\text { disponibilização nos sistemas } \\
\text { de saúde } 53 \text {. }\end{array}$ \\
\hline $\begin{array}{l}\text { Profilaxia pós- } \\
\text { exposição (PEP) }\end{array}$ & $\begin{array}{l}\text { Uso de ARV por } 28 \\
\text { dias pós-exposição } \\
\text { com parceiros } \\
\text { infectados e carga } \\
\text { viral detectável } \\
\text { ou com sorologia } \\
\text { desconhecida. }\end{array}$ & $\begin{array}{c}\text { 89\%, considerando } \\
\text { experimentos em } \\
\text { não humanos } 54 \text {. }\end{array}$ & $\begin{array}{l}\text { Último recurso preventivo para } \\
\text { pessoas não infectadas pelo } \\
\text { HIV com relações com potencial } \\
\text { exposição. } \\
\text { Usado como complemento e não } \\
\text { substituição de métodos 55. }\end{array}$ & $\begin{array}{l}\text { Conhecimento é baixo, mesmo entre } \\
\text { profissionais de saúde e população } \\
\text { com risco substancial de infecção } \\
\text { pelo HIV } 56 \text {. } \\
\text { Uso é baixo, mesmo entre } \\
\text { aqueles que conhecem ou têm } \\
\text { o medicamento para início da } \\
\text { profilaxia } 57 \text {. } \\
\text { Impacto na epidemia é reduzido } \\
\text { (uso mais frequente em pessoas não } \\
\text { expostas ao HIV) 58. } \\
\text { Altas taxas de não adesão, 31\% 59. } \\
\text { Aconselhamento não aumentou a } \\
\text { adesão } 59 \text {. }\end{array}$ \\
\hline $\begin{array}{l}\text { Práticas não } \\
\text { penetrativas }\end{array}$ & $\begin{array}{l}\text { Manejo das práticas } \\
\text { sexuais de forma a } \\
\text { evitar penetração, } \\
\text { especialmente } \\
\text { anal e vaginal. } \\
\text { Também conhecido } \\
\text { como gouinage, } \\
\text { o termo francês } \\
\text { originalmente } \\
\text { se referia a } \\
\text { mulheres lésbicas, } \\
\text { mas atualmente } \\
\text { descreve práticas } \\
\text { sexuais entre } \\
\text { homens que não } \\
\text { envolvem } \\
\text { penetração anal. }\end{array}$ & $\begin{array}{l}\text { Evita a exposição. } \\
\text { Em tese, é } \\
\text { completamente } \\
\text { seguro. }\end{array}$ & $\begin{array}{c}\text { Dispensa insumos preventivos, } \\
\text { sendo, portanto, acessível a todas } \\
\text { as pessoas e em todas } \\
\text { as ocasiões. } \\
\text { Risco de transmissão baixo ou } \\
\text { inexistente, a depender das } \\
\text { práticas que envolvem. } \\
\text { Como práticas preventivas } \\
\text { desenvolvidas no âmbito } \\
\text { comunitário, têm alto grau de } \\
\text { aceitação e uso em contextos } \\
\text { específicos } 60 \text {. }\end{array}$ & $\begin{array}{l}\text { Para algumas pessoas, podem não } \\
\text { ser suficientemente satisfatórias. } \\
\text { A intenção de não penetração pode } \\
\text { se modificar no decorrer da relação } \\
\text { sexual, sem que os praticantes } \\
\text { tenham acesso a outras opções } \\
\text { preventivas imediatas. }\end{array}$ \\
\hline
\end{tabular}

(continua) 


\begin{tabular}{|c|c|c|c|c|}
\hline $\begin{array}{l}\text { Método } \\
\text { preventivo }\end{array}$ & Uso & Proteção & Vantagens & Desvantagens \\
\hline $\begin{array}{l}\text { Práticas } \\
\text { soroadaptativas }\end{array}$ & $\begin{array}{l}\text { Acordos sexuais } \\
\text { baseados no } \\
\text { conhecimento da } \\
\text { sorologia ou da } \\
\text { carga viral entre os } \\
\text { parceiros. Exemplos } \\
\text { são seleção de } \\
\text { parceiros pela } \\
\text { sorologia e escolhas } \\
\text { de práticas menos } \\
\text { arriscadas quando } \\
\text { há sorodiscordância } \\
\text { conhecida } 61 \text {. }\end{array}$ & $\begin{array}{l}\text { Acréscimo de } 53 \% \text { de } \\
\text { proteção para o HIV } \\
\text { e } 14 \% \text { para outras } \\
\text { IST nas relações } \\
\text { homossexuais em } \\
\text { comparação com } \\
\text { adoção de nenhum } \\
\text { método preventivo } \\
62 \text {. }\end{array}$ & $\begin{array}{c}\text { Aumenta segurança em situações de } \\
\text { impossibilidade ou escolha de não } \\
\text { usar outros métodos. } \\
\text { Pessoas que sabem estar infectadas } \\
\text { pelo HIV são significativamente mais } \\
\text { propensas a tomar medidas para } \\
\text { proteger os seus } \\
\text { parceiros } 63,64 \text {. }\end{array}$ & $\begin{array}{l}\text { Comparado ao uso do preservativo, } \\
\text { aumenta o risco em 79\% para o HIV e } \\
61 \% \text { para as IST } 62 . \\
\text { Dependente das condições para a } \\
\text { revelação do diagnóstico pela parceria; } \\
\text { contextos discriminatórios inibem a } \\
\text { revelação 62,65,66. } \\
\text { Em contexto de maior incidência do HIV, } \\
\text { pode haver número elevado de pessoas } \\
\text { que não sabem estar com uma infecção } \\
\text { recente } 62,65,66 \text {. } \\
\text { Contexto de baixa adesão ao ARV limita } \\
\text { o uso de informação sobre carga } \\
\text { viral } 62,65,66 .\end{array}$ \\
\hline $\begin{array}{l}\text { Preservativo } \\
\text { masculino }\end{array}$ & $\begin{array}{l}\text { Uso de preservativo } \\
\text { masculino em todas } \\
\text { as relações } 67 .\end{array}$ & 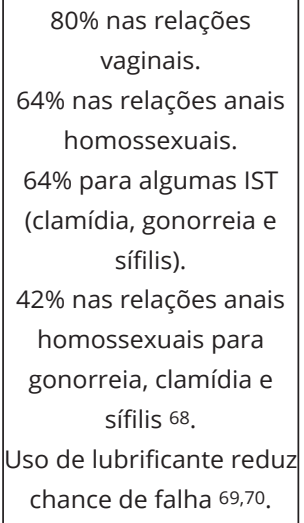 & $\begin{array}{c}\text { Boa aceitação, acesso gratuito ou } \\
\text { a baixo custo, e poucos efeitos } \\
\text { adversos. } \\
\text { Previne também gravidez. }\end{array}$ & $\begin{array}{l}\text { Quando consideradas todas as } \\
\text { relações sexuais, a frequência de uso } \\
\text { é baixa } 71 \text {. } \\
\text { Não reduz risco quando usado } \\
\text { “algumas vezes" para } \\
\text { homossexuais } 72 \text {. } \\
\text { Alta frequência de uso incorreto e de } \\
\text { inadequação do produto: utilização } \\
\text { apenas no momento da ejaculação } \\
\text { (20-60\%); rompimentos e vazamentos } \\
\text { (25-40\%), inadequação de tamanho } \\
\text { (45\%) 73,74. }\end{array}$ \\
\hline $\begin{array}{l}\text { Preservativo } \\
\text { feminino }\end{array}$ & $\begin{array}{l}\text { Uso de preservativo } \\
\text { feminino em todas } \\
\text { as relações. }\end{array}$ & $\begin{array}{l}\text { Taxa de proteção } \\
\text { similar à do } \\
\text { preservativo } \\
\text { masculino } 75,76 \text {. }\end{array}$ & $\begin{array}{c}\text { Favorece autonomia para das } \\
\text { mulheres } 77 . \\
\text { Aumenta práticas seguras e diminui } \\
\text { a incidência de IST } 78 . \\
\text { Previne também gravidez. }\end{array}$ & $\begin{array}{c}\text { Baixa disponibilidade. } \\
\text { Baixo uso, de } 1 \% 79 \text { a } 3,5 \% 80 . \\
\text { Boa aceitação, mas a continuidade } \\
\text { do uso depende de estratégias de } \\
\text { aconselhamento e } \\
\text { treinamento continuadas } 77 . \\
\text { Alto custo } 81,82,83 \text {. }\end{array}$ \\
\hline $\begin{array}{l}\text { Tratamento } \\
\text { como prevenção }\end{array}$ & $\begin{array}{c}\text { Uso de ARV } \\
\text { por pessoas } \\
\text { infectadas por HIV, } \\
\text { independentemente } \\
\text { do CD4 e com carga } \\
\text { viral indetectável } 21 \text {. }\end{array}$ & $\begin{array}{c}\text { 96\% nas relações } \\
\text { com pares } \\
\text { sorodiferente. }\end{array}$ & $\begin{array}{l}\text { Associado a benefícios para saúde } \\
\text { das pessoas infectadas. } \\
\text { Alta motivação de uso (deixar de } \\
\text { transmitir o vírus). } \\
\text { Método de maior impacto estimado } \\
\text { no controle da epidemia } 84 \text {. } \\
\text { Em relações sorodiferentes, é } \\
\text { preferido em relação à PrEP. }\end{array}$ & $\begin{array}{l}\text { Elevadas taxas de não adesão } 85 . \\
\text { Pessoas infectadas podem não } \\
\text { querer iniciar o tratamento } 86 . \\
\text { Profissionais da saúde não acreditam } \\
\text { na efetividade do método }{ }^{87} \text {. } \\
\text { Barreiras estruturais dificultam } \\
\text { acesso e continuidade do } \\
\text { tratamento. }\end{array}$ \\
\hline
\end{tabular}

ARV: antirretrioviral; IST: infecções sexualmente transmissíveis; TDF: tenofir; TDF/FTC: tenofovir associado à entricitabina. 
em relações estáveis. A PEP, por sua vez, complementa estratégias baseadas no preservativo, pós-rompimento ou quando não utilizado.

A gestão do risco sexual parte do pressuposto de que as pessoas são cotidianamente sujeitos de suas práticas sexuais e que quase sempre conviverão com algum grau de risco nas suas práticas sexuais 45. Pessoas em busca de PrEP, em geral, convivem com graus de risco maiores do que a média populacional, mas isso não significa que não se preocupam com o HIV ou IST. Ao contrário: alguém que busca esse método é, fundamentalmente, alguém que está buscando formas de minimizar riscos, sem que isso signifique, necessariamente, mudar substantivamente as formas como experimenta seus desejos e prazeres sexuais. É importante notar, no entanto, que pode ocorrer um estranhamento do profissional de saúde diante dos relatos de práticas e situações de exposições ao HIV. Contudo, cabe ao profissional desenvolver uma postura de diálogo orientada para o sucesso prático da prevenção na vida daquele indivíduo a quem está atendendo. A prevenção combinada oferece precisamente tal vantagem: se diferentes métodos podem ser utilizados em diferentes situações, então há mais possibilidades para conjugar preferências sexuais e prevenção, sem que um método seja considerado superior aos outros.

No que se refere à flexibilidade de agendamento e complementaridade de uma equipe multiprofissional, os projetos demonstrativos de PrEP indicaram um padrão de uso dos serviços caracterizado por faltas e procura de consultas extras para a resolução de demandas, como IST, suspeita de infecção por HIV ou retirada de medicamento, quando ele está acabando. A irregularidade na frequência pode comprometer a oferta de atendimentos, particularmente consultas médicas, e aumentar o risco de usuários não receberem medicamentos regularmente. Diante dessa realidade, uma abordagem pragmática e não punitiva pode favorecer a vinculação com o serviço e, ao mesmo tempo, evitar que as faltas e as demandas não planejadas desorganizem a rotina do serviço. Ações que podem contribuir incluem a previsão de espaços nas agendas para atender às intercorrências, adoção de lembretes de consultas enviados pelos serviços aos usuários e a criação de estratégias específicas para acompanhamento de faltosos.

\section{Lacuna entre a estrutura dos serviços e os protocolos de oferta de PrEP}

Análises iniciais da implantação da PrEP nos serviços participantes do Estudo Combina! mostraram relativa incompatibilidade entre os protocolos clínicos de pesquisa - que se assemelham às diretrizes do Ministério da Saúde - e a estrutura existente nos serviços. Em ambulatórios, houve menor disponibilidade de aconselhadores e menor familiaridade das equipes com estratégias preventivas; enquanto nos serviços de testagem houve restrição de médicos, ausência de procedimentos de seguimento do usuário, uma vez que são serviços organizados para atendimentos de curto seguimento, e dificuldades de acesso aos exames laboratoriais para monitoramento da segurança de uso da PrEP.

Independentemente do tipo de serviço, observou-se duplicação de procedimentos e de abordagens entre as consultas de aconselhamento, médicas e farmacêuticas. Houve, também, reações negativas de parte dos profissionais acerca da PrEP, como não acreditar na segurança ou na efetividade do método; atribuir ao preservativo e a outros métodos clássicos um maior grau de proteção e dificuldades para lidar com relatos de não uso do preservativo concomitante à PrEP. Quase metade dos profissionais disse acreditar que não teriam tempo para atender aos usuários de PrEP. Como resultado, em alguns serviços, a quantidade de profissionais envolvidos com a PrEP foi insuficiente, o número de usuários iniciando PrEP foi baixo, o tempo para a início da profilaxia superior a trinta dias e os atendimentos excessivamente demorados, superiores a duas horas. A sobreposição propiciou que usuários questionassem a pertinência das consultas e julgassem tal protocolo inadequado. Ao longo do tempo, isso pode gerar desmotivação para a continuidade da profilaxia, assim como sobrecarregar agendas já escassas. Dessa maneira, considera-se que a ampliação e o fortalecimento da rede de serviços também precisam estar acompanhados de uma revisão de protocolos clínicos de modo a simplificar procedimentos e racionalizar os atendimentos, especialmente em relação à PEP e testagem anti-HIV.

Adicionalmente, projetos demonstrativos mostraram alta aceitação do método, mas a busca pela profilaxia esteve fortemente circunscrita a gays e HSH, com maior renda e grau de informação, enquanto outras populações mais vulneráveis, como mulheres trabalhadoras do sexo, jovens negros e pessoas transexuais não chegaram aos serviços de PrEP. Logo, mesmo entre grupos contemplados 
nas diretrizes de oferta de PrEP, será necessário redobrar a força de iniciativas para incluir populações em maior vulnerabilidade social.

Há de se lembrar ainda que o principal desafio é promover um amplo acesso à PrEP, o que torna três aspectos centrais para o êxito: (1) aumentar a cobertura dos serviços; (2) assegurar que os serviços sejam ambientes culturalmente diversos e livres de discriminação e (3) investir em intervenções comunitárias, incluindo as redes sociais em que as pessoas possam contar com suas relações de suporte para acesso à informação e referência aos serviços.

\section{Evidências sobre o uso da PrEP que precisam ser produzidas}

Do ponto de vista da política nacional para a oferta de PrEP no SUS, dois aspectos merecem destaque. Primeiro, por falta de evidências, alguns grupos com alta prevalência do HIV não foram incluídos nas atuais diretrizes nacionais. É o caso de adolescentes, usuários de drogas e heterossexuais com maior exposição ao HIV. Estudos de efetividade em adolescentes são limitados em todo o mundo. Até 2020, estudos demonstrativos brasileiros deverão prover dados de efetividade nesse grupo, assim como marcos referenciais e legais para que tal segmento possa acessar a PrEP sem necessidade de consentimento parental. É importante notar que no Brasil existe vasta legislação para amparar a oferta de PrEP para adolescentes 46 . No caso específico de heterossexuais, faltam evidências para caracterizar subpopulações que poderiam se beneficiar da PrEP. Estudos de comportamento sexual, bem como de aceitação, uso e custo-benefício do método envolvendo usuários de serviços de IST e PEP poderiam contribuir para reduzir esta lacuna. No caso dos usuários de drogas, o principal desafio seria identificar serviços que poderiam ofertar a PrEP de forma segura, sobretudo para usuários de crack, que apresentam prevalência superior a da população geral ${ }^{14}$. Infelizmente, no país, são quase inexistentes serviços de redução de danos.

\section{Considerações finais e recomendações}

No presente ensaio, buscou-se mostrar que os potenciais benefícios da PrEP estão circunscritos às características contextuais e às necessidades particulares de indivíduos com maior risco de infecção. O método contribui para superar importantes dificuldades que limitam o uso consistente de outros métodos, sobretudo o do preservativo, e pode, assim, gerar um importante impacto no enfrentamento da epidemia. Todavia, por ser um método serviço-dependente, seu êxito depende da capacidade de os serviços adotarem uma organização do trabalho centrada nas necessidades dos usuários, tanto objetivas quanto subjetivas. Procurou-se demonstrar, ainda, que o enfrentamento de desafios que a introdução da PrEP tem suscitado - como promover a adesão e lidar com as IST - se dará no contexto dos serviços. Notadamente, esforços mais intensos deverão ser dispensados para incluir em PrEP segmentos mais vulneráveis ao HIV.

Nesse sentido, é importante considerar que a PrEP pode ser uma janela de oportunidade para o enfrentamento da epidemia, em um cenário marcado por mudanças significativas no plano cultural, expressas na construção de novas relações de gênero e de exercício da sexualidade e da prática sexual, especialmente em novas gerações. Isso tem influenciado, por exemplo, a forma como pessoas encontram parcerias, estabelecem acordos sexuais e redefinem as possibilidades de prevenção. Nesse cenário, especificamente em relação à tecnologia, pode-se destacar o crescente uso, sobretudo entre HSH, de aplicativos georreferenciados para encontro de parcerias, nos quais se delineiam possibilidades de práticas sexuais e preventivas, escolha de parceria em função de, por exemplo, sorologia para HIV, possibilidade de sexo com parcerias externas a um relacionamento, assim como de uso de PrEP 47.

É possível, dessa forma, que se esteja em um período de novas configurações de expressão sexual com as quais as estratégias de prevenção e a PrEP precisam dialogar para que, por um lado, não percam sua atualidade e aplicabilidade e, por outro, atuem como um elemento consolidador das novas configurações culturais e sexuais.

Isso já tem ocorrido de forma mais explícita com o exemplo anteriormente citado, no qual os aplicativos têm sido reconhecidos como uma aceitável fonte de informação sobre saúde sexual $48 \mathrm{e}$ como forma de promover maior motivação para que indivíduos se vinculem aos serviços e a PrEP 49. 
Por último, nenhum método preventivo por si só será suficiente para o controle efetivo da epidemia. A ampliação das opções preventivas, com a incorporação da PrEP e de outros métodos baseados nos antirretrovirais na perspectiva da prevenção combinada podem significar passos largos nessa direção, desde que conjugados à qualificação de profissionais e serviços e intervenções estruturais propiciadoras do acesso e da adesão.

\section{Colaboradores}

E. M. Zucchi e A. Grangeiro participaram da concepção, redação, interpretação e revisão final do texto. D. Ferraz, T. F. Pinheiro e T. Alencar colaboraram na redação, interpretação e revisão final do texto. L. Ferguson contribuiu na interpretação e revisão final do texto. D. L. Estevam e R. Munhoz contribuíram na revisão final do texto.

\section{Outros membros da equipe do Estudo Combina!}

Gina Ardila, Ana Cecilia Bueno, Lorruan Alves, Jackeline Oliveira Gomes, Geovana Gomes, Lis Neves, Carolina Brasileiro, Erico Arruda, Renata Moraes, Alan Silveira, Juliane Cardoso, Bruno Kauss, Andrea Fachel Leal, Claudia Marnatti.

\section{Agradecimentos}

Especialmente aos participantes do estudo, pesquisadores de campo e profissionais dos serviços colaboradores do Estudo Combina!. À Secretaria de Vigilância em Saúde, Ministério da Saúde; Organização das Nações Unidas para a Educação, a Ciência e a Cultura (UNESCO) e Conselho Nacional de Desenvolvimento Científico e Tecnológico (CNPq).

\section{Referências}

1. Anderson PL, Kiser JJ, Gardner EM, Rower JE, Meditz A, Grant RM. Pharmacological considerations for tenofovir and emtricitabine to prevent $\mathrm{HIV}$ infection. J Antimicrob Chemother 2011; 66:240-50.

2. Grangeiro A, Ferraz D, Calazans G, Zucchi EM, Díaz-Bermúdez XP. O efeito dos métodos preventivos na redução do risco de infecção pelo HIV nas relações sexuais e seu potencial impacto em âmbito populacional: uma revisão da literatura. Rev Bras Epidemiol 2015; 18 Suppl 1:43-62.

3. Chang LW, Serwadda D, Quinn TC, Wawer MJ, Gray RH, Reynolds SJ. Combination implementation for HIV prevention: moving from clinical trial evidence to population-level effects. Lancet Infect Dis 2013; 13:65-76.

4. Kippax S, Stephenson N. Beyond the distinction between biomedical and social dimensions of HIV prevention through the lens of a social public health. Am J Public Health 2012; 102:789-99.

5. Fonner VA, Dalglish SL, Kennedy CE, Baggaley R, O’Reilly KR, Koechlin FM, et al. Effectiveness and safety of oral HIV pre-exposure prophylaxis (PrEP) for all populations: a systematic review and meta-analysis. AIDS 2016; 30:197383.

6. Ravasi G, Grinsztejn B, Baruch R, Guanira JV, Luque R, Cáceres CF, et al. Towards a fair consideration of PrEP as part of combination HIV prevention in Latin America. J Int AIDS Soc 2016; 19(7 Suppl 6):21113.

7. Zablotska I, Grulich AE, Phanuphak N, Anand T, Janyam S, Poonkasetwattana M, et al. PrEP implementation in the Asia-Pacific region: opportunities, implementation and barriers. J Int AIDS Soc 2016; 19 (7 Suppl 6):21119.

8. Jenness SM, Goodreau SM, Rosenberg E, Beylerian EN, Hoover KW, Smith DK, et al. Impact of the Centers for Disease Control's HIV Preexposure prophylaxis guidelines for men who have sex with men in the United States. J Infect Dis 2016; 214:1800-7.

9. Secretaria de Ciência, Tecnologia e Insumos Estratégicos, Ministério da Saúde. Portaria no 21 , de 25 de maio de 2017. Torna pública a decisão de incorporar o tenofovir associado a entricitabina (TDF/FTC 300/200mg) como profilaxia pré-exposição (PrEP) para populações sob maior risco de adquirir o vírus da imunodeficiência humana (HIV), no âmbito do Sistema Único de Saúde - SUS. Diário Oficial da União 2017; 29 mai. 
10. Hoagland B, Moreira RI, De Boni RB, Kallas EG, Madruga JV, Vasconcelos R, et al. High preexposure prophylaxis uptake and early adherence among men who have sex with men and transgender women at risk for HIV infection: the PrEP Brasil demonstration project. J Int AIDS Soc 2017; 20:21472.

11. Grangeiro A, Couto MT, Peres MF, Luiz O, Zucchi EM, Castilho EA, et al. Pre-exposure and postexposure prophylaxes and the combination HIV prevention methods (The Combine! study): protocol for a pragmatic clinical trial at public healthcare clinics in Brazil. BMJ Open 2015; 5:e009021.

12. World Health Organization. Guideline on when to start antiretroviral therapy and on preexposure prophylaxis for HIV. Geneva: World Health Organization; 2015.

13. Departamento de Vigilância, Prevenção e Controle das Infecções Sexualmente Transmissíveis, do HIV/AIDS e das Hepatites Virais, Secretaria de Vigilância em Saúde, Ministério da Saúde. Protocolo clínico e diretrizes terapêuticas para profilaxia pré-exposição (PrEP) de risco à infecção pelo HIV. Brasília: Ministério da Saúde; 2017.

14. Bertoni N, Burnett C, Cruz MS, Andrade T, Bastos FI, Leal E, et al. Exploring sex differences in drug use, health and service use characteristics among young urban crack users in Brazil. Int J Equity Health 2014; 13:70.

15. Starks TJ, Payton G, Golub SA, Weinberger CL, Parsons JT. Contextualizing condom use: Intimacy Interference, stigma, and unprotected sex. J Health Psychol 2014; 19:711-20.

16. Ministério da Saúde. Pesquisa de conhecimentos, atitudes e práticas na população brasileira. Brasília: Ministério da Saúde; 2016.

17. Ayres JRCM, Paiva V, França Jr. I. Conceitos e práticas de prevenção: da história natural da doença ao quadro da vulnerabilidade e direitos humanos. In: Paiva V, Ayres JRCM, Buchalla C, organizadores. Vulnerabilidade e direitos humanos: prevenção e promoção da saúde. Curitiba: Juruá Editora; 2012. p. 71-94.

18. Sullivan PS, Salazar L, Buchbinder S, Sanchez TH. Estimating the proportion of HIV transmissions from main sex partners among men who have sex with men in five US cities. AIDS 2009; 23:1153-62.

19. Patel P, Borkowf CB, Brooks JT, Lasry A, Lansky A, Mermin J. Estimating per-act HIV transmission risk: a systematic review. AIDS 2014; 28:1509-19.

20. Goodreau SM, Carnegie NB, Vittinghoff E, Lama JR, Sanchez J, Grinsztejn B, et al. What drives the US and Peruvian HIV epidemics in men who have sex with men (MSM)? PLoS One 2012; 7:e50522.

21. Cohen MS, Chen YQ, McCauley M, Gamble T, Hosseinipour MC, Kumarasamy N, et al. Prevention of HIV-1 infection with early antiretroviral therapy. N Engl J Med 2011; 365:493-505.

22. Molina JM, Charreau I, Spire B, Cotte L, Chas J, Capitant C, et al. Efficacy, safety, and effect on sexual behaviour of on-demand pre-exposure prophylaxis for HIV in men who have sex with men: an observational cohort study. Lancet HIV 2017; 4:e402-10.
23. Liu AY, Cohen SE, Vittinghoff E, Anderson PL, Doblecki-Lewis S, Bacon O, et al. HIV pre-exposure prophylaxis integrated with municipal and community based sexual health services. JAMA Int Med 2016; 176:75-84.

24. Grant RM, Lama JR, Anderson PL, McMahan V, Liu AY, Vargas L, et al. Preexposure chemoprophylaxis for HIV prevention in men who have sex with men. N Engl J Med 2010; 363:2587-99.

25. Grant RM, Anderson PL, McMahan V, Liu A, Amico KR, Mehrotra M, et al. Uptake of preexposure prophylaxis, sexual practices, and HIV incidence in men and transgender women who have sex with men: a cohort study. Lancet Infect Dis 2014; 14:820-9.

26. McCormack S, Dunn DT, Desai M, Dolling D, Gafor M, Gilson R, et al. Pre-exposure prophylaxis to prevent the acquisition of HIV-1 infection (PROUD): effectiveness results from the pilot phase of a pragmatic open-label randomised trial. Lancet 2016; 387:53-60.

27. Volk JE, Marcus JL, Phengrasamy T, Blechinger D, Nguyen DP, Follansbee S, et al. No new HIV infections with increasing use of HIV preexposure prophylaxis in a clinical practice setting. Clin Infect Dis 2015; 61:1601-3.

28. Baeten JM, Donnell D, Ndase P, Mugo NR, Campbell JD, Wangisi J, et al. Antiretroviral prophylaxis for HIV prevention in heterosexual men and women. N Engl J Med 2012; 367:399410.

29. Donnell D, Baeten JM, Bumpus NN, Brantley J, Bangsberg DR, Haberer JE, et al. HIV protective efficacy and correlates of tenofovir blood concentrations in a clinical trial of PrEP for HIV prevention. J Acquir Immune Defic Syndr 2014; 66:340-8.

30. Hosek SG, Landovitz RJ, Kapogiannis B, Siberry GK, Rudy B, Rutledge B, et al. Safety and feasibility of antiretroviral preexposure prophylaxis for adolescent men who have sex with men aged 15 to 17 years in the United States. JAMA Pediatr 2017; 171:1063-71.

31. Oldenburg CE, Nunn AS, Montgomery M, Almonte A, Mena L, Patel RR, et al. Behavioral changes following uptake of hiv pre-exposure prophylaxis among men who have sex with men in a clinical setting. AIDS Behav 2018; 22:1075-9.

32. Solomon MM, Lama JR, Glidden DV, Mulligan $\mathrm{K}, \mathrm{McMahan}$ V, Liu AY, et al. Changes in renal function associated with oral emtricitabine/ tenofovir disoproxil fumarate use for HIV preexposure prophylaxis. AIDS 2014; 28:851-9.

33. Girometti N, Gutierrez A, Nwokolo N, McOwan A, Whitlock G. High HIV incidence in men who have sex with men following an early syphilis diagnosis: is there room for pre-exposure prophylaxis as a prevention strategy? Sex Transm Infect 2017; 93:320-2.

34. Scott HM, Klausner JD. Sexually transmitted infections and pre-exposure prophylaxis: challenges and opportunities among men who have sex with men in the US. AIDS Res Ther 2016; 13:5. 
35. Hotton AL, Gratzer B, Mehta SD. Association between serosorting and bacterial sexually transmitted infection among HIV-negative men who have sex with men at an urban lesbian, gay, bisexual, and transgender health center. Sex Transm Dis 2012; 39:959-64.

36. Arnold T, Brinkley-Rubinstein L, Chan PA, Perez-Brumer A, Bologna ES, Beauchamps L, et al. Social, structural, behavioral and clinical factors influencing retention in pre-exposure prophylaxis (PrEP) care in Mississippi. PLoS One 2017; 12:e172354.

37. Yi S, Tuot S, Mwai GW, Ngin C, Chhim K, Pal $\mathrm{K}$, et al. Awareness and willingness to use HIV pre-exposure prophylaxis among men who have sex with men in low- and middle-income countries: a systematic review and meta-analysis. J Int AIDS Soc 2017; 20:21580.

38. Goparaju L, Praschan NC, Warren-Jeanpiere L, Experton LS, Young MA, Kas-Saye S. Stigma, partners, providers and costs: potential barriers to prep uptake among US women. J AIDS Clin Res 2017; 8:730.

39. Calabrese SK, Underhill K. How stigma surrounding the use of hiv preexposure prophylaxis undermines prevention and pleasure: a call to destigmatize "truvada whores". Am J Public Health 2015; 105:1960-4.

40. Ferraz DAS, Nemes MIB. Avaliação da implantação de atividades de prevenção das DST/ AIDS na atenção básica: um estudo de caso na Região Metropolitana de São Paulo, Brasil. Cad Saúde Pública 2009; 25 Suppl 2:S240-50.

41. Ayres JRCM. Cuidado: trabalho e interação nas práticas de saúde. Rio de Janeiro: Centro de Estudos e Pesquisa em Saúde Coletiva, Instituto de Medicina Social, Universidade Federal do Rio de Janeiro/ABRASCO; 2009.

42. Madureira VSF, Trentini M. Da utilização do preservativo masculino à prevenção de DST/ AIDS. Ciênc Saúde Coletiva 2008; 13:1807-16.

43. Campos GWS, Amaral MA. A clínica ampliada e compartilhada, a gestão democrática e redes de atenção como referenciais teórico-operacionais para a reforma do hospital. Ciênc Saúde Coletiva 2007; 12:849-59.

44. Pupo LR, Ayres JRCM. Contribuições e limites do uso da abordagem centrada na pessoa para a fundamentação teórica do aconselhamento em DST/AIDS. Temas Psicol (Online) 2013; 21:1107-24.

45. Ferraz D, Paiva V. Sexo, direitos humanos e AIDS: uma análise das novas tecnologias de prevenção do HIV no contexto brasileiro. Rev Bras Epidemiol 2015; 18 Suppl 1:89-103.

46. Centro de Referência e Treinamento DST/ AIDS, Coordenação Estadual de DST/AIDS, Secretaria Estadual de Saúde. Prevenção das DST/AIDS em adolescentes e jovens: brochuras de referência para os profissionais de saúde. São Paulo: Secretaria Estadual de Saúde; 2007.

47. Goedel WC, Duncan DT. Geosocial-networking app usage patterns of gay, bisexual, and other men who have sex with men: survey among users of Grindr, a mobile dating app. JMIR Public Health Surveill 2015; 1:e4.
48. Sun CJ, Stowers J, Miller C, Bachmann LH, Rhodes SD. Acceptability and feasibility of using established geosocial and sexual networking mobile applications to promote HIV and STD testing among men who have sex with men. AIDS Behav 2015; 19:543-52.

49. Fuchs JD, Stojanovski K, Vittinghoff E, McMahan VM, Hosek SG, Amico KR. A mobile health strategy to support adherence to antiretroviral preexposure prophylaxis. AIDS Patient Care STDS 2018; 32:104-11.

50. Okwundu CI, Uthman OA, Okoromah CA. Antiretroviral pre-exposure prophylaxis (PrEP) for preventing HIV in high-risk individuals. Cochrane Database Syst Rev 2012; (7):CD007189

51. Van Damme L, Corneli A, Ahmed K, Agot K, Lombaard J, Kapiga S, et al. Preexposure prophylaxis for HIV infection among African women. N Engl J Med 2012; 367:411-22.

52. Marrazzo JM, Ramjee G, Richardson BA, Gomez K, Mgodi N, Nair G, et al. Tenofovir-based preexposure prophylaxis for HIV infection among African women. N Engl J Med 2015; 372:509-18.

53. Juusola JL, Brandeau ML, Owens DK, Bendavid E. The cost-effectiveness of preexposure prophylaxis for HIV prevention in men who have sex with men in the United States. Ann Intern Med 2012; 156:541-50.

54. Irvine C, Egan KJ, Shubber Z, Van Rompay KK, Beanland RL, Ford N. Efficacy of HIV postexposure prophylaxis: systematic review and meta-analysis of nonhuman primate studies. Clin Infect Dis 2015; 60 Suppl 3:S165-9.

55. Korner H, Hendry O, Kippax S. Safe sex after post-exposure prophylaxis for HIV: intentions, challenges and ambivalences in narratives of gay men. AIDS Care 2006; 18:879-87.

56. Rodríguez A, Castel AD, Parish CL, Willis S, Feaster DJ, Kharfen M, et al. HIV medical providers' perceptions of the use of antiretroviral therapy as non-occupational post- exposure prophylaxis (nPEP) in two major metropolitan areas. J Acquir Immune Defic Syndr 2013; 64 Suppl 1:S68-79.

57. Schechter M, do Lago RF, Mendelsohn AB, Moreira RI, Moulton LH, Harrison LH, et al. Behavioral impact, acceptability, and HIV incidence among homosexual men with access to postexposure chemoprophylaxis for HIV. J Acquir Immune Defic Syndr 2004; 35:519-25.

58. Poynten IM, Smith DE, Cooper DA, Kaldor JM, Grulich AE. The public health impact of widespread availability of nonoccupational postexposure prophylaxis against HIV. HIV Med 2007; 8:374-81.

59. Oldenburg CE, Bärnighausen T, Harling G, Mimiaga MJ, Mayer KH. Adherence to postexposure prophylaxis for non-forcible sexual exposure to HIV: a systematic review and metaanalysis. AIDS Behav 2014; 18:217-25.

60. Dubois-Arber F, Jeannin A, Lociciro S, Balthasar H. Risk reduction practices in men who have sex with men in Switzerland: serosorting, strategic positioning, and withdrawal before ejaculation. Arch Sex Behav 2012; 41:1263-72. 
61. McConnell JJ, Bragg L, Shiboski S, Grant RM. Sexual seroadaptation: lessons for prevention and sex research from a cohort of HIV-positive men who have sex with men. PLoS One 2010; 5:e8831.

62. Kennedy CE, Bernard LJ, Muessig KE, Konda KA, Akl EA, Lo YR, et al. Serosorting and HIV/ STI infection among HIV-negative MSM and transgender people: a systematic review and meta-analysis to inform WHO guidelines. J Sex Transm Dis 2013; 2013:583627.

63. Higgins DL, Galavotti C, O’Reilly KR, Schnell DJ, Moore M, Rugg DL, et al. Evidence for the effects of HIV antibody counseling and testing on risk behaviors. JAMA 1991; 266:2419-29.

64. Hays RB, Paul J, Ekstrand M, Kegeles SM, Stall R, Coates TJ. Actual versus perceived HIV status, sexual behaviors and predictors of unprotected sex among young gay and bisexual men who identify as HIV-negative, HIV-positive and untested. AIDS 1997; 11:1495-502.

65. Eaton LA, Kalichman SC, O'Connell DA, Karchner WD. A strategy for selecting sexual partners believed to pose little/no risks for HIV: serosorting and its implications for HIV transmission. AIDS Care 2009; 21:1279-88.

66. O'Connell A, Reed S, Serovich J. The efficacy of serostatus disclosure for HIV transmission risk reduction. AIDS Behav 2015; 19:283-90.

67. Holmes KK, Levine R, Weaver M. Effectiveness of condoms in preventing sexually transmitted infections. Bull World Health Organ 2004; 82:454-61.

68. World Health Organization. Guidelines: prevention and treatment of HIV and other sexually transmitted infections among men who have sex with men and transgender people: recommendations for a public health approach. Geneva: World Health Organization; 2011.

69. Dezzutti CS, Brown ER, Moncla B, Russo J, Cost M, Wang L, et al. Is wetter better? An evaluation of over-the-counter personal lubricants for safety and anti-HIV-1 activity. PLoS One 2012; 7:e48328.

70. Gorbach PM, Weiss RE, Fuchs E, Jeffries RA, Hezerah M, Brown S, et al. The slippery slope: lubricant use and rectal sexually transmitted infections: a newly identified risk. Sex Transm Dis 2012; 39:59-64.

71. Hearst N, Chen S. Condom promotion for AIDS prevention in the developing world: is it working? Stud Fam Plann 2004; 35:39-47.

72. Smith DK, Herbst JH, Zhang X, Rose CE. Condom effectiveness for HIV prevention by consistency of use among men who have sex with men in the United States. J Acquir Immune Defic Syndr 2015; 68:337-44.

73. Crosby R. State of condom use in HIV prevention science and practice. Curr HIV/AIDS Rep 2013; 10:59-64.

74. Sanders SA, Yarber WL, Kaufman EL, Crosby RA, Graham CA, Milhausen RR. Condom use errors and problems: a global view. Sex Health 2012; 9:81-95.
75. Gallo MF, Kilbourne-Brook M, Coffey PS. A review of the effectiveness and acceptability of the female condom for dual protection. Sex Health 2012; 9:18-26.

76. Dowdy DW, Sweat MD, Holtgrave DR. Country-wide distribution of the nitrile female condom (FC2) in Brazil and South Africa: a costeffectiveness analysis. AIDS 2006; 20:2091-8.

77. Núcleo de Estudos de População, Universidade Estadual de Campinas; Associação Brasileira Interdisciplinar de Aids; Fundo de População das Nações Unidas. Preservativo feminino: das políticas globais à realidade brasileira. Brasília: Fundo de População das Nações Unidas; 2011.

78. French PP, Latka M, Gollub EL, Rogers C, Hoover DR, Stein ZA. Use-effectiveness of the female versus male condom in preventing sexually transmitted disease in women. Sex Transm Dis 2003; 30:433-9.

79. Ferraz DAS, Grangeiro A. Mulheres brasileiras: sexualidade e prevenção da AIDS. In: Venturi G, Godinho T, organizadores. Mulheres brasileiras e gênero nos espaços público e privado. São Paulo: SESC/Editora Fundação Perseu Abramo; 2013. p. 172-84.

80. Santos N, Filipe EMV, Paiva V, Buchalla CM, Bergamaschi DP. Saúde reprodutiva e sexualidade em mulheres HIV positivas. J Bras Aids 2003; 4:29-37.

81. Beksinska ME, Piaggio G, Smit JA, Wu J, Zhang Y, Pienaar J, et al. Performance and safety of the second-generation female condom (FC2) versus the Woman's, the VA worn-of-women, and the Cupid female condoms: a randomised controlled non-inferiority crossover trial. Lancet Glob Health 2013; 3:e146-52.

82. Gollub EL. The female condom: tool for women's empowerment. Am J Public Health 2000; 90:1377-81.

83. Vijayakumar G, Mabude Z, Smit J, Beksinska M, Lurie M. A review of female-condom effectiveness: patterns of use and impact on protected sex acts and STI incidence. Int J STD AIDS 2006; 17:652-9.

84. Cremin I, Alsallaq R, Dybul M, Piot P, Garnett $\mathrm{G}$, Hallett TB. The new role of antiretrovirals in combination HIV prevention: a mathematical modelling analysis. AIDS 2013; 27:447-58.

85. Gardner EM, McLees MP, Steiner JF, Del Rio C, Burman WJ. The spectrum of engagement in HIV care and its relevance to test-and-treat strategies for prevention of HIV infection. Clin Infect Dis 2011; 52:793-800.

86. Charurat ME, Emmanuel B, Akolo C, Keshinro B, Nowak RG, Kennedy S, et al. Uptake of treatment as prevention for HIV and continuum of care among HIV-positive men who have sex with men in Nigeria. J Acquir Immune Defic Syndr 2015; 68 Suppl 2:S114-23.

87. Evans C, Bennett J, Croston M, Brito-Ault N, Bruton J. "In reality, it is complex and difficult": UK nurses' perspectives on "treatment as prevention" within HIV care. AIDS Care 2015; 27:753-7. 


\section{Abstract}

Pre-exposure prophylaxis (PrEP) has been considered a promising strategy for controlling the global HIV epidemic. However, it is necessary to translate the knowledge accumulated from clinical trials and demosntration studies to the reality of health services and the groups most vulnerable to infection in order to achieve broad coverage with PrEP. The article proposes a reflection on this challenge, focusing on three dimensions: users of prophylaxis, with an emphasis on the contexts of sexual practices and the potential exposures to $H I V ;$ the advantages of prophylaxis as compared to other methods and the challenges for protective and safe use; and health services, considering the organizational principles to ensure greater success in the supply and incorporation of PrEP as part of combination prevention strategies. The following principles were analyzed: uniqueness of care, freedom of choice and non-hierarchization of prevention methods, sexual risk management, scheduling flexibility, and complementary and multidisciplinary care. These principles can foster organization of the health service and care, facilitating linkage and retention in care. Some comments were offered on the relative incompatibility between the existing structure of services and the Brazilian Ministry of Health guidelines for offering PrEP. The conclusion was that the success of PrEP as a public health policy depends on two essential factors: ensuring that health services are culturally diverse settings, free of discrimination, and the intensification of community-based interventions, including social networks, in order to reduce inequalities in access to PrEP and health services as a whole.

Pre-Exposure Prophylaxis; HIV; Sexual Health; Health Services

\section{Resumen}

La profilaxis pre-exposición sexual (PrEP) ha sido considerada una estratégica y prometedora acción en el control de la epidemia de VIH globalmente. No obstante, se hace necesario trasladar el conocimiento acumulado por los estudios de eficacia, que muestren la realidad de los servicios y la población más vulnerable a la infección, de manera que se alcance una amplia cobertura de la PrEP. Se propone una reflexión sobre este desafío centrándose en dos dimensiones: usuarios de profilaxis, con énfasis en contextos de prácticas y potencial exposición al VIH; ventajas comparativas de la profilaxis, respecto a los demás métodos, y los desafios para un uso con protección y seguro; y los servicios, considerando principios organizativos que podrían otorgar un mayor éxito a la oferta e incorporación de la PrEP, en el seno de estrategias de prevención combinada. Se analizaron como algunos principios: singularidad en el cuidado, autonomía para la elección y no jerarquización de los métodos, gestión del riesgo sexual y flexibilidad de citas y complementariedad de un equipo multiprofesional. Todos ellos pueden favorecer la organización del servicio y del cuidado, facilitando la vinculación y preservación de los usuarios. Se elaboraron algunas consideraciones, acerca de una relativa incompatibilidad entre la estructura existente en los servicios y las directrices del Ministerio de Salud de Brasil para la oferta de PrEP. Se concluyó que el mayor éxito de la PrEP, como política pública de salud, depende de dos aspectos centrales: asegurar que los servicios sean ambientes culturalmente diversos y libres de discriminación y la intensificación de las intervenciones comunitarias, incluyendo redes sociales, de forma que se reduzcan las inequidades en el acceso a los servicios y al PrEP.

Profilaxis Pre-Exposición; VIH; Salud Sexual; Servicios de Salud
Recebido em 26/Nov/2017

Versão final reapresentada em 10/Abr/2018 Aprovado em 02/Mai/2018 